\title{
Interventional Cardiology: Volume 9, Issue 6
}

\section{Introduction}

Heart disease comprises of both coronary as well as valvular disease. However, coronary heart disease is more common of the two, and results in the death of more than 370,000 individuals every year. Interventional cardiology is a subspecialty of cardiology, which deals with the diagnosis and treatment of a variety of vascular and cardiac disorders in a minimally invasive manner using cardiac catheterization. Cardiac catheterization involves the insertion of flexible tubes through the skin, and then threading them through the blood vessels in order to resolve any blockage in the vessel. Interventional cardiology has significantly contributed to our understanding of coronary diseases by allowing an understanding of the correlation between the clinical picture of coronary disease and the underlying pathology of the disease. The better understanding of the fundamental mechanisms underlying coronary disease will aid the management and/or prevention of coronary disease in the coming decades.

The journal, Interventional Cardiology deals with all aspects of the subject, with special focus on the latest advancements in the field. The current issue of Interventional Cardiology presents some interesting studies. Chu et al. [1], developed a new ECG algorithm for identifying the location of the accessory pathway (AP) in WolffParkinson-White syndrome (WPW) patients. Ranocchi et al. [2], presented the successful management of an ischemic ventricular septal defect (VSD) caused due to the rupture of the posterior left ventricular free-wall, following blockage of a right coronary artery (RCA). Chu et al. [3], tested the accuracy of a newly developed algorithm for a 12-lead electrocardiogram which was directed at identifying the accessory pathways (APs) in the WolffParkinson-White (WPW) syndrome. Cicek [4] compared the long-term clinical outcomes of durable polymer drug-eluting stent (DP-DES) with biodegradable polymer drug eluting stents (BP-DES) in patients suffering from right coronary artery (RCA) chronic total occlusion (CTO). Carini et al. [5], described a case of an aorto-pulmonary window identified during a surgery for Type A interrupted aortic arch, which was repaired without any hindrance. Kraisornsawat et al. [6], conducted a literature review with special focus on caring for pediatric patients with cardiac catheterization suffering from vaso-vagal reflex. Nunokawa [7] authored a review on the creation of a native arteriovenous fistula (AVF) for the purpose of hemodialysis (HD), and the factors to be considered while proceeding for the same

The electrical activity in the human heart is initiated when the cardiac action potential originates in the sinoatrial (SA) node. From there, the electrical stimulus is transmitted to the atrioventricular (AV) node through the internodal pathways, where it stays for a short duration. From the AV node, the stimulus spreads through the bundle of His to the Purkinje fibers and the endocardium located at the apex of the heart, and then finally it spreads to the ventricular myocardium. The Wolff-Parkinson-White syndrome (WPW) is a condition where in addition to the AV node, an accessory pathway known as the bundle of Kent acts as a communicating door between the atria and the ventricles. This accessory pathway (AP) does not have the same rate-slowing properties as the AV node, and might conduct electrical activity at a higher rate than the AV node. Chu et al. [1], developed a new ECG algorithm for identifying the location of AP in WPW patients.

Ranocchi et al. [2], have presented the successful management of an ischemic ventricular septal defect (VSD) caused due to the rupture of the posterior left ventricular free-wall, following blockage of a right coronary artery (RCA). The VSD was repaired by using gelatin-resorcinolformaldehyde glue (GRF) along with three
Ian B. A. Menown *

Craigavon Cardiac Centre, Queen's University, Belfast, UK *Author for correspondence:

ian.menown@southerntrust.hscni.net

Submitted: 13 December 2017

Accepted: 14 December 2017

Published online: 19 December 2017 
pericardial patches. Extracorporeal membrane oxygenation (ECMO) and intra-aortic balloon pump (IABP) were used to decrease the tension in the left ventricle (LV) wall, and for reducing the risk of recurrence of VSD and free wall rupture.

Chu et al. [3], tested the accuracy of a newly developed algorithm for a 12-lead electrocardiogram which was directed at identifying the accessory pathways (APs) in the Wolff-Parkinson-White (WPW) syndrome. The new algorithm based electrocardiogram was tested in 109 patients. It was observed that the new algorithm aided the localization of AP in the WPW patients with high accuracy.

A drug-eluting stent (DES) is a peripheral which when placed into the diseased coronary arteries, slowly releases a drug that blocks cell proliferation. The first generation drug eluting stents (DESs) have considerably reduced the requirement for repeated revascularization as compared to bare metal stents (BMS). In order to improve the first generation DES, newer devices using either biocompatible durable polymer blended with metal alloys or biodegradable polymers blended with stainless steel have been developed. The biodegradable polymer drug eluting stents (BP-DES) developed to overcome the limitations associated with the first generation durable polymer DES (DP-DES), have not exhibited consistent results in clinical trials. Cicek [4] compared the long-term clinical outcomes of DP-DES with BPDES in patients suffering from right coronary artery (RCA) chronic total occlusion (CTO). The results of the study revealed that the efficacy of BP-DES is comparable to that of DP-DES.

Aorto-pulmonary window is a rare congenital defect of the heart in which a connection (window) is present between the aorta and the pulmonary artery. This opening allows for the oxygenated blood to enter the pulmonary artery from the aorta, at high pressure, resulting in too much blood flow to the lungs. This condition occurs in $0.1 \%-0.2 \%$ of the patients suffering from congenital cardiac disease. Aortopulmonary window can also occur in conjunction with other heart defects such as: atrial septal defect, tetralogy, truncus arteriosus, pulmonary atresia, interrupted aortic arch, and patent ductus arteriosus. Carini et al. [5], described a case of an aortopulmonary window identified during a surgery for Type A interrupted aortic arch, which was repaired without any hindrance.

Vaso-vagal reflex is a common complication in pediatric patients undergoing cardiac catheterization. In order to understand the vaso-vagal reflex among pediatric patients with cardiac catheterization, Kraisornsawat et al. [6], conducted a literature review with special focus on caring for such patients.
End stage renal disease (ESRD) is the final stage of chronic kidney disease (CKD); it is the time when renal transplant or dialysis is necessary to stay alive. Even though, renal transplant is the best treatment strategy for patients suffering from end-stage renal disease (ESRD), most of the ESRD patients opt for hemodialysis (HD) to manage their condition. A wellfunctioning and safe vascular access (VA) is the cornerstone of an efficient HD procedure. Three main types of VA are: (1) native arteriovenous fistula (AVF), (2) arteriovenous graft, and (3) central venous catheter (CVC). However, AVF is the first VA of choice for chronic HD; it is the best VA for longevity and has the lowest level of association with morbidity and mortality. Therefore, the use of AVF is strongly recommended by guidelines across the world. The high frequency of primary failure is the major complication associated with AVF; primary AVF failure has been reported in up to $40 \%$ of the cases following AVF placement. There are many factors known to be associated with the high rate of AVF failure; old age, peripheral vascular disease (PVD), and coronary artery disease $(\mathrm{CAD})$ are all known to be the clinical indicators correlated with an increased AVF failure risk. Nunokawa [7] has authored a review on the creation of an AVF, and the factors to be considered while proceeding for the same.

\section{References}

1. Chu SD, Pham KQ, Tran DV. 12 Lead electrocardiogram algorithm for the localization of accessory pathways using simple parameters in patients with typical Wolff-ParkinsonWhite syndrome. Interv. Cardiol. 9(6): 229-237 (2017).

2. Ranocchi F, Piciche M, Cottini M, et al. Inferior ischemic ventricular septal defect: Atypical triple pericardial patch implantation technique. Interv. Cardiol. 9(6): 239-242 (2017).

3. Chu SD, Pham KQ, Tran DV. Accuracy of the new electrocardiogram algorithm in predicting localization of accessory pathways in patients with typical Wolff-ParkinsonWhite syndrome. Interv. Cardiol. 9(6): 243-249 (2017).

4. Cicek D. Durable polymer versus bioabsorbable polymer drug eluting stents in right coronary artery chronic total occlusions: Eighteen months (median) results of a single-center experience. Interv. Cardiol. 9(6): 251-257 (2017).

5. Carini L, Cantarano MS, Lombardi M, et al. A rare case of an unexpected aortopulmonary window. Interv. Cardiol. 9(6): 259-261 (2017).

6. Kraisornsawat T, Usahawong U, Salapsri S. Vaso-vagal reflex in paediatric patients with cardiac catheterization: Integrative literature review. Interv. Cardiol. 9(6): 267-269 (2017).

7. Nunokawa M. Creation of primary arteriovenous fistula:Vascular access for hemodialysis. Interv. Cardiol. 9(6): 271-279 (2017). 